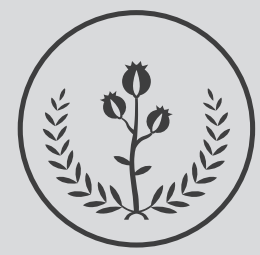

FUCS

\section{Rer per

\title{
Intoxicación accidental por ácido acético glacial en niños
}

Gloria Marcela Preciado Correal MDa Hernando Andrés Olaya-Acosta MDa

Accidental glacial acetic acid intoxication in children

${ }^{a}$ Toxicología Clínica. Fundación Universitaria de Ciencias de la Salud, Bogotá DC, Colombia.

\section{R E S U M E N}

Introducción: el ácido acético en bajas concentraciones se utiliza como vinagre para los alimentos, al que le da el sabor amargo y olor característico. También es un reactivo importante para la producción de otros compuestos orgánicos. Cuando está concentrado tiene usos industriales, como es el ácido acético glacial (99\%) el cual se ingirió en forma accidental en este caso. Presentación del caso: paciente de 8 años de edad quien tras posterior trauma cráneo encefálico leve es manejado en casa con ácido acético glacial que ingirió accidentalmente con posterior odinofagia, emesis e intolerancia a la vía oral. Hubo lesiones iniciales en faringe y amígdalas manejadas con el respectivo antibiótico. Al no haber mejoría consulta de nuevo por aumento del compromiso faríngeo, evidenciándose quemadura y lesión esofágica por cáusticos con estenosis. El seguimiento a los 6 meses, después de realizar dilataciones por medio de esofagoduodenoscopia, el paciente tuvo buena evolución clínica.

Palabras clave: ácido acético, cáustico, estenosis esofágica, intoxicación, vinagre.

(C) 2022 Fundación Universitaria de Ciencias de la Salud - FUCS. Este es un artículo Open Access bajo la licencia CC BY-NC-ND (http://creativecommons.org/licenses/by-nc-nd/4.0/).

\section{ABSTRACT}

Introduction: low concentrations of acetic acid are commonly used as table top condiment giving a bitter taste featuring a characteristic smell. It is also an important reactive to make other organic compounds. Case report: at high concentrations it has industrial uses, such as $99 \%$ glacial acetic acid which was ingested by an 8 -year-old patient in this case. Patient was managed at home after a mild head trauma with glacial acetic acid which he ingested accidentally presenting with pain and

\section{INFORMACIÓN DEL ARTÍCULO}

Historia del artículo:

Fecha recibido: octubre 4 de 2018

Fecha aceptado: noviembre 19 de 2019
Autor para correspondencia.
Dra. Marcela Preciado gmpreciado@fucsalud.edu.co
DOI

10.31260/RepertMedCir.01217372.222 
difficulty in swallowing and emesis. The initial pharyngeal and tonsil lesions were managed with the respective antibiotic therapy. He consults again for not presenting improvement and increase of the pharyngeal compromise, evidencing an esophageal burn and a corrosive injury with stenosis. At 6-month follow-up, after undergoing dilations through esophagogastroduedenoscopy, patient presented good clinical progression.

Key words: acetic acid, caustic, esophageal stenosis, intoxication, vinegar.

(C) 2022 Fundación Universitaria de Ciencias de la Salud - FUCS. This is an open access article under the CC BY-NC-ND license (http://creativecommons.org/licenses/by-nc-nd/4.0/).

\section{IN T RODUCCIÓN}

La posibilidad de que los niños estén expuestos a sustancias tóxicas es una eventualidad frecuente por diferentes motivos, la prevención no se puede alcanzar en su totalidad, en especial en cuanto a disminuir la incidencia que tiene la ingestión de productos caústicos, sobre todo en los países en desarrollo como es el nuestro. No se puede establecer una epidemiologia clara ya que no siempre se reportan, en su mayoría son accidentales por error en el uso de sustancias o por ignorancia, curiosidad o falta de conocimiento.

La ingestión de agentes cáusticos, como el ácido acético o la soda caustica, pueden conducir a problemas complicados como la necrosis, perforación y estenosis de las vías digestivas. Hay sustancias ácidas o alcalinas que causan complicaciones graves como la necrosis de licuefacción. Las cantidades que ingieren los menores pueden ser grandes debido a que no tienen una noción clara del peligro, causando necrosis, gastritis severa, síntomas respiratorios por ácidos volátiles o lesiones a nivel de vía área superior, como es este caso. ${ }^{1}$

Se ha avanzado en el tratamiento de este tipo de accidentes al establecer el grado de compromiso mediante endoscopia digestiva alta, tomografía computarizada y ultrasonido, pero el diagnóstico debe siempre sospecharse desde la clínica para realizar el tratamiento oportuno y así prevenir complicaciones a corto y largo plazo. El caso que se describe a continuación es importante y se quiere dar a conocer para prevenir posibles diagnósticos tardíos de intoxicación por cáusticos.

\section{REPORTE DE CASO}

Paciente de 8 años de edad quien en casa sufre un trauma craneoencefálico leve con posterior dolor en sitio de hematoma subgaleal, utilizando como analgésico el ácido acético como remedio casero. En este caso se utilizó ácido acético glacial que fue ingerido $(5 \mathrm{cc})$ presentando odinofagia y malestar gástrico. Consultó inicialmente al servicio de salud donde se diagnosticó rinofaringitis viral; a los tres días acudió a nuestra institución por presentar múltiples episodios eméticos, hiporexia, episodios de somnolencia, astenia, adinamia y persistencia de odinofagia. Al examen físico se evidencia quemadura en faringe con formación de membranas secundarias, se indica valoración por el servicio de toxicología clínica y cirugía pediátrica (figura 1), donde realizan esofagoduodenoscopia observando placas blanquecinas en paladar blando, amígdalas palatinas y orofaringe, que ocupan toda la circunferencia y comprometen la totalidad del esófago, sin perforaciones ni áreas de necrosis considerando quemadura esofágica grado IIB. Se procede a dejar al paciente con catéter central, sin vía oral, nutrición parenteral total, protección gástrica, analgésico, antibiótico y corticoide. Permanece con este manejo intrahospitalario por 8 días y el control de la endoscopia revela zonas de cicatrización acompañadas de membranas blanquecinas escasas en la totalidad de la longitud del esófago. Inicialmente no se observan zonas estenóticas que impidan el paso del endoscopio; la mucosa de la cámara gástrica no muestra alteraciones, dándose egreso de la institución. El seguimiento ambulatorio ha requerido dilataciones esofágicas durante 6 meses.

El manejo de las lesiones por cáusticos está ampliamente descrito y se decide seguirlo de acuerdo con el diagnóstico como lo indica la literatura, sin vía oral, antibióticos y corticoides parenterales, los cuales son controversiales, y continuar el seguimiento por medio de esofagoduodenoscopia. ${ }^{2}$

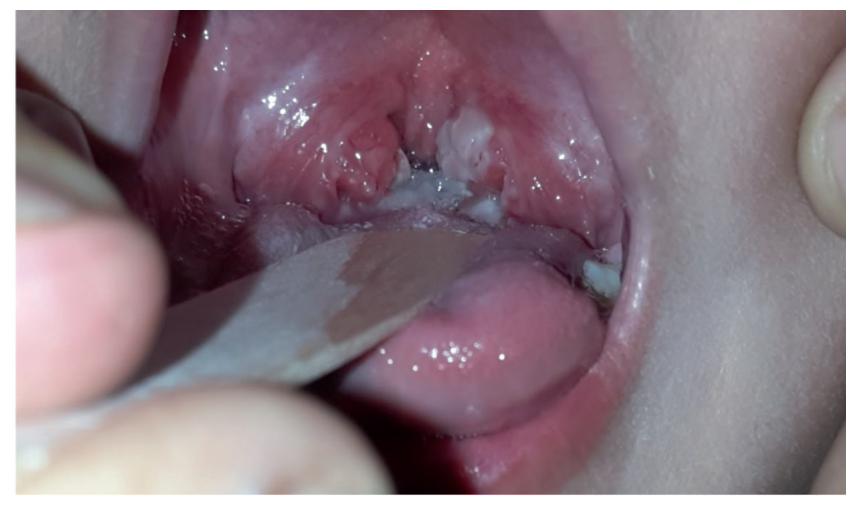

Figura 1. Placas en la faringe secundarias a quemadura por ácido acético glacial. Fuente: los autores. 


\section{RESULTADOS Y SEGUIMIENTO}

El paciente fue manejado con antiemético (ondansetrón $4 \mathrm{mg}$ cada 8 horas por el antecedente de alergia a metoclopramida), antibióticos (1.3 gr ampicilina/ sulbactam IV cada 6 horas) y analgésico, paracetamol $400 \mathrm{mg}$ IV cada 6 horas por 8 días intrahospitalario. La endoscopia de vías digestivas superiores evidencia placas eritematosas en faringe, senos piriformes y vallécula, hay ulceración circunferencial con exudado perilesional de $5 \mathrm{~cm}$ sin signos de sangrado activo y a nivel del estómago se observa gastritis antral y duodeno sano. Conclusión: quemadura de tercio distal de esófago grado IIB y gastritis antral. Se pide valoración por cirugía pediátrica, para descartar complicación del cuadro descrito. Se solicita radiografía de vías digestivas altas encontrándose estenosis esofágica en tercio distal. Se inició manejo médico con corticoesteroides sistémicos en las primeras 24 horas para disminuir el riesgo de estenosis esofágica y se iniciaron dilataciones por esofagoduodenoscopia.

\section{DISCUSIÓN}

La intoxicación por cáusticos no intencional en población pediátrica es un problema de salud pública global, que a pesar de la legislación para limitar las concentraciones de estos materiales y hacer contenedores a prueba de niños, sigue manteniéndose como un motivo de consulta frecuente en los servicios de urgencias, dada la costumbre de reenvasar estas sustancias en contenedores de uso habitual para los niños. Como consecuencia de la ingesta se producen quemaduras en el tracto gastrointestinal, lo cual puede llevar a múltiples complicaciones como la estenosis esofágica e incluso la muerte.

La mayoría de los autores recomiendan la evaluación temprana para evaluar las lesiones esofágicas, permitiendo dar un tratamiento oportuno y previniendo las complicaciones tardías secundarias a la exposición de cáusticos. Se han descrito múltiples tratamientos para el manejo de estas lesiones, entre ellos el uso de esteroides; la utilidad de éstos es un tema controversial, ya que inhiben la respuesta inflamatoria, lo que en teoría previene la estenosis esofágica, sin embargo existe evidencia de que no hay diferencia estadística entre el manejo temprano con esteroides y el expectante. ${ }^{2,3}$

En la búsqueda de la literatura se encontraron pocos estudios integrativos entre los cuales no había ningún metaanálisis, con una calidad de evidencia aceptable. El objetivo de llegar a un consenso para la prevención primaria de complicaciones secundarias a la ingesta de cáusticos no es clara. ${ }^{4,5}$ En este caso, el paciente tuvo adecuada evolución clínica y respuesta al manejo médico realizado.

\section{CONFLICTOS DE INTERÉS}

Los autores declaran no tener ningún conflicto de interés.

\section{REFEREN CIAS}

1. Khan S, Orenstein SR. Ingestion. In: Kliegman RM, St Geme JW, Blum NJ, Shah SS, Tasker RC, Wilson KM, editors. Nelson Textbook of Pediatrics. 20 ed. Philadelphia: Elsevier; 2016. p. 1793-6.

2. Bird JH, Kumar S, Paul C, Ramsden JD. Controversies in the management of caustic ingestion injury: an evidence-based review. Clinical otolaryngology : official journal of ENT-UK ; official journal of Netherlands Society for Oto-Rhino-Laryngology \& Cervico-Facial Surgery. 2017;42(3):701-8. doi: https://doi. org/10.1080/15563650701285420

3. Fulton JA, Hoffman RS. Steroids in second degree caustic burns of the esophagus: a systematic pooled analysis of fifty years of human data: 1956-2006. Clin Toxicol (Phila). 2007;45(4):402-8. doi: https://doi.org/10.1080/15563650701285420

4. Shub MD. Therapy of caustic ingestion: new treatment considerations. Curr Opin Pediatr. 2015;27(5):609-13. doi: https:// doi.org/10.1097/MOP.0000000000000257

5. De Lusong MAA, Timbol ABG, Tuazon DJS. Management of esophageal caustic injury. World J Gastrointest Pharmacol Ther. 2017;8(2):90-8. doi: https://doi.org/10.4292/wjgpt.v8.i2.90 